Pacific Journal of Mathematic 


\title{
CONVEXITY PROPERTIES OF INTEGRAL MEANS OF ANALYTIC FUNCTIONS
}

\author{
H. SHNIAD
}

1. Introduction. Let $f=f(z)$ denote an analytic function of the complex variable $z$ in the open circle $|z|<R$. For each positive number $t$, the mean of order $t$ of the modulus of $f(z)$ is defined as follows:

$$
\mathbb{M}_{t}(r ; f)=\left[\frac{1}{2 \pi} \int_{0}^{2 \pi}\left|f\left(r e^{i \theta}\right)\right|^{t} d \theta\right]^{1 / t}, \quad(0 \leq r<R) .
$$

The reader might consult $[5$, p. 143-144; 3 ; and 4, p. 134-146] for some of the properties of this mean value function $\mathfrak{M}_{t}(r ; f)$.

We consider the question: does the analyticity in $|z|<R$ of the function $f$ imply the convexity of the mean $\mathbb{M}_{t}(r ; f)$ as a function of $r$ in the interval $0 \leq r<$ $R$ ? It is known [ 1 ] that:

(A) Unless the function $f$ is suitably restricted, the set of positive values $t$ for which the question may be answered affirmatively has a finite upper bound.

(B) If the number $t$ is of the form $2 / k$, with $k$ a positive integer, then, for every analytic function $f$, the mean of order $t$ is convex.

(C) If the function $f$ vanishes at the origin, then the mean $\mathbb{M}_{t}(r ; f)$ is convex for every fixed positive number $t$.

(D) If the function $f$ has no zero in the circle, then its mean of order $t$ is convex, provided that the positive number satisfies $t \leq 2$.

(E) If the function $f$ has at most $k$ zeros, $k \geqq 1$, in the circle, then the mean of order $t$ is convex provided that the positive number $t$ satisfies $t \leqq 2 / k$.

The main purpose of this paper is to prove that, for every analytic function $f$, the mean of order four is convex. Moreover, we show by example that if the number $t$ is greater than 5.66, then there is an analytic function whose mean of order $t$ is not convex.

2. Means of nonvanishing functions. Assume that $g(z)$ is analytic in $|z|<R$,

Received August 10, 1952.

Pacific J. Math. 3 (1953), 657-666 
and that the expansion for $g(z)$ in the given circle is

$$
g(z)=\sum_{n=0}^{\infty} a_{n} z^{n} .
$$

Then the integral

$$
h(r ; g)=\frac{1}{2 \pi} \int_{0}^{2 \pi}\left|g\left(r e^{i \theta}\right)\right|^{2} d \theta
$$

has the expansion

$$
h(r ; g)=\sum_{n=0}^{\infty}\left|a_{n}\right|^{2} r^{2 n},
$$

valid in $r<R$. Let

$$
Q(r ; g, c)=h h^{\prime \prime}-c\left(h^{\prime}\right)^{2},
$$

where primes denote differentiation with respect to $r, h$ is the function $h(r ; g)$, and $c$ is a constant independent of the variable $r$ and of the function $g$. If $C$ is a class of functions $\{g(z)\}$, and if, for all functions $g$ in this class $C$, for all $r<R$, and for a particular positive value $c_{0}$, the inequality

$$
Q\left(r ; g, c_{0}\right) \geq 0
$$

holds, then the inequality

$$
Q(r ; g, c) \geq 0
$$

holds for all $c<c_{0}$, all $r<R$, and all functions $g$ in the class $C$. We now specify the class $C$ to be the class of all functions $g(z)$ which are analytic and do not vanish in $|z|<R$. If $f(z)$ is in class $C$, then any single-valued branch of $[f(z)]^{\alpha}$ where $\alpha$ is an arbitrary real number, is also in class $C$. Given a function $f_{0}(z)$ in class $C$, and a fixed positive number $t$, let $g_{0}(z)$ be a single-valued branch of $\left[f_{0}(z)\right]^{t / 2}$; and let

$$
h_{0}(r)=\frac{1}{2 \pi} \int_{0}^{2 \pi}\left|g_{0}(z)\right|^{2} d \theta
$$

Then

$$
\mathbb{M}_{t}\left(r ; f_{0}\right)=\left[h_{0}\right]^{1 / t}
$$


and since $h_{0}$ is a nonvanishing function of $r$, we have

$$
\frac{d^{2} M_{t}\left(r ; f_{0}\right)}{d r^{2}}=P \cdot Q\left[r ; g_{0},(1-1 / t)\right] \text {, }
$$

where

$$
P=\frac{M_{t}\left(r ; f_{0}\right)}{t h_{0}^{2}}>0
$$

Every function $g(z)$ in class $C$ is a single-valued branch of $[f(z)]^{t / 2}$, where $f(z)$ is some appropriate function in class $C$. Therefore, for positive values $t$, the mean $\mathbb{M}_{t}(r ; f)$ is a convex function of $r$ for all functions $f$ in class $C$ if and only if

$$
Q[r ; g,(1-1 / t)] \geq 0
$$

for all functions $g$ in class $C$. Since the inequality $1-1 / t<1-1 / t_{0}$ holds for all $t$ and $t_{0}$ satisfying $0<t<t_{0}$, we conclude from the preceding remarks that, if the positive value $t_{0}$ is such that the mean $\mathbb{N}_{t_{0}}(r ; f)$ is convex for all nonvanishing $f(z)$, then the mean $\mathfrak{M}_{t}(r ; f)$ is convex for all nonvanishing $f(z)$, provided that $t$ is any positive value not exceeding $t_{0}$.

For a simple example of a function $\dddot{M}_{t}(r ; f)$ which is not convex, consider the mean of order eight of a single-valued branch of

$$
f(z)=\sqrt{1+z} \quad \text { in }|z|<1 .
$$

In this case, we have

$$
h(r)=1+4 r^{2}+r^{4}
$$

and $[h(r)]^{1 / 8}$ is not convex in $0 \leq r<1$.

Since, for every analytic function $f$, the mean of order two is convex, it now follows that there exists a greatest positive value $t_{0}$, in the range $2 \leq t_{0}<8$, such that $\mathfrak{M}_{t_{0}}(r ; t)$ is convex for all nonvanishing analytic functions. It will be a corollary of our result that this greatest value $t_{0}$ satisfies the inequalities $4 \leq t_{0}<5.66$

3. Preliminary lemmata. The proof of our main theorem will be based on the following lemmata.

LEMMA 1. Let $a_{i}(i=1,2, \ldots)$ be a sequence of positive numbers such 
that the sum

$$
\sum_{i=1}^{\infty} 1 / a_{i}
$$

converges to the finite value $M$. If the sequence of real variables $x_{i}(i=1,2, \ldots)$ is restricted to satisfy the inequality

$$
\sum_{i=1}^{\infty} a_{i} x_{i}^{2} \leq B
$$

then the maximum value of the function

$$
f=\sum_{i=1}^{\infty} x_{i}
$$

is $(B M)^{1 / 2}$.

Proof. We consider first maximizing

$$
f_{n}=\sum_{i=1}^{n} x_{i},
$$

with the variables subject to the condition

$$
\sum_{i=1}^{n} a_{i} x_{i}^{2}=B
$$

Let

$$
M_{n}=\sum_{i=1}^{n} 1 / a_{i} .
$$

The critical points of the function $f_{n}$ are at the solutions of the simultaneous equations

$$
a_{i} x_{i}=a_{j} x_{j} \quad(i, j=1, \cdots, n),
$$

which are given by 


$$
x_{i}^{2}=B\left(M_{n} a_{i}^{2}\right), \quad(i=1, \cdots, n) .
$$

Therefore, the maximum $f_{n}$ is $M_{n}\left(B / M_{n}\right)^{1 / 2}$ or $\left(B M_{n}\right)^{1 / 2}$. Since $M_{n}<M$, and all the values $a_{i}$ are positive, it follows that for all $n$ the partial sums $f_{n}$ are bounded by $(B M)^{1 / 2}$ and the conclusion of the lemma follows.

Lемма 2. Let $S$ be the sum

$$
S=\sum_{n=3}^{\infty} 1 /\left(6 n^{2}-9 n+2\right)
$$

Then this sum $S$ is less than 0.09504.

Proof. The function $f(n)=1 /\left(6 n^{2}-9 n+2\right)$ has the following expansion in powers of $1 /(n-1)$ :

$$
f(n)=\sum_{k=2}^{\infty} a_{k} /(n-1)^{k},
$$

with $a_{2}=1 / 6, a_{3}=-1 / 12, a_{4}=5 / 72$. For determining subsequent values of $a_{k}$, it is convenient to use the recursion formula:

$$
a_{k+2}=\left(a_{k}-3 a_{k+1}\right) / 6
$$

The coefficients $a_{2}$ and $a_{3}$ are positive and negative respectively. Therefore it follows directly from the recursion formula that the general coefficients $a_{k}$ alternate in sign. By another use of the recursion formula, we see that the sum $a_{k}+$ $a_{k+1}$ is equal to $\left(a_{k-2}-a_{k-1}\right) / 12$, and therefore that the sign of the sum $a_{k}+$ $a_{k+1}$ is the same as that of the coefficient $a_{k-2}$, or of the coefficient $a_{k}$. Since the inequalities $\left|a_{2}\right|>\left|a_{3}\right|>\left|a_{4}\right|$ hold, it now follows that the numerical values of the coefficients all decrease with increasing $k$. Let $\zeta(k)$ be the Riemann zeta-function, and let $s(k)=\zeta(k)-1$. Since the foregoing expansion for $f(n)$ is an absolutely convergent series, the sum $S$ may be expanded in an alternating series of the form

$$
S=\sum_{k=2}^{\infty} a_{k} s(k),
$$

whose terms decrease in numerical value with increasing $k$. Using ( see [2]) the approximations $s(2)=0.644935, s(4)=0.082324, s(6)=0.017344, s(8)=$ $0.004078, s(10)=0.000995$, which are too large, and the approximations $s(3)=$ 
$0.202056, s(5)=0.036927, s(7)=0.008349, s(9)=0.002008$, which are too small, we obtain the value 0.09504 stated in the lemma by summing this last series up to and including the term for $k=10$.

LEMMA 3. Let

$$
y=\sqrt{x}+\sqrt{0.04752} \sqrt{9 x^{2}-10 x+1},
$$

where $x$ lies in the range $0 \leq x \leq 1 / 9$. Then the maximum value of $y$ is less than $(\sqrt{2}-1)$.

Proof. Setting the derivative of $y$ equal to zero, we find that the value of $x$ maximizing $y$ is the solution of the equation

$$
0.04752 x(10-18 x)^{2}-\left(9 x^{2}-10 x+1\right)=0 .
$$

This critical value of $x$ lies between 0.07 and 0.08 . Therefore

$$
\begin{aligned}
\max y<\sqrt{0.08}+\sqrt{0.04752\left[9(0.07)^{2}-10(0.07)+1\right]} & \\
& <0.283+0.129=0.412 .
\end{aligned}
$$

Since $(\sqrt{2}-1)$ is greater than 0.414 , the conclusion of the lemma follows.

4. The mean of order four. Let

$$
g(z)=[f(z)]^{2}
$$

have the expansion

$$
g(z)=\sum_{n=0}^{\infty} a_{n} z^{n},
$$

valid in $|z|<R$. Following the ideas developed in $\$ 2$, we see that

$$
\mathfrak{M}_{4}(r ; f)=[h(r)]^{1 / 4},
$$

with

$$
h(r)=\sum_{n=0}^{\infty}\left|a_{n}\right|^{2} r^{2 n},
$$

and that $\mathbb{M}_{4}(r ; f)$ is convex in $r<R$ if and only if 


$$
Q(r) \equiv h h^{\prime \prime}-\frac{3}{4}\left(h^{\prime}\right)^{2}=\sum_{i, j=0}^{\infty} Q_{i j} p_{i} p_{j} r^{2(i+j)-2}
$$

with

$$
Q_{i j}=i(2 i-1)+j(2 j-1)-3 i j \text { and } p_{i}=\left|a_{i}\right|^{2}
$$

is nonnegative in the interval $0 \leqq r<R$. The only coefficient $Q_{i j}$ which is negative is $Q_{11}=-1$. That the mean of order four is convex may be concluded from the following theorem.

THEOREM. If a function $g(z)$ is analytic in the circle $|z|<R$, and the function

$$
\left[\frac{1}{2 \pi} \int_{0}^{2 \pi}\left|g\left(r e^{i \theta}\right)\right|^{2} d \theta\right]^{1 / 4}
$$

is not convex as a function of $r$ in the interval $r<R$, then $g(z)$ is not the square of an analytic function in $|z|<R$.

Proof. It is pointed out in the introduction that if $f(0)=0$, then the mean $\mathbb{P}_{t}(r ; f)$ is convex for all $t$. Therefore we may assume that

$$
[f(0)]^{2}=g(0)=p_{0}
$$

is not zero. The hypothesis of the theorem implies that

$$
Q(r)=\sum_{i, j=0}^{\infty} Q_{i j} p_{i} p_{j} r^{2(i+j)-2}
$$

takes on negative values; since $Q_{11}$ is the only negative coefficient, this is possible only if the value $p_{1}=\left|a_{1}\right|^{2}$ is not zero. Therefore, we may make the normalizations

$$
a_{0}=1, a_{1}=\sqrt{2}, p_{0}=1 \text {, and } p_{1}=2 \text {. }
$$

Let

$$
\begin{aligned}
Q_{1}(r)= & 2 p_{0} p_{1}+\left(12 p_{0} p_{2}-p_{1}{ }^{2}\right) r^{2}+2 p_{1} p_{2} r^{4} \\
& +2 \sum_{n=3}^{\infty}\left(Q_{0 n} p_{0} p_{n} r^{2 n-2}+Q_{1 n} p_{1} p_{n} r^{2 n}\right),
\end{aligned}
$$


with $Q_{0 n}=n(2 n-1)$ and $Q_{1 n}=2 n^{2}-4 n+1$. Since $Q(r) \geq Q_{1}(r)$, and $Q_{1}(r)$ can be negative only for values of $r$ satisfying

$$
2 p_{0} p_{1}-p_{1}^{2} r^{2}<0
$$

we have in the normalized case the result that $Q_{1}(r)$ is negative for some $r>1$; and the expression

$$
Q_{2}(r)=4+\left(12 p_{2}-4\right) r^{2}+\left[4 p_{2}+\sum_{n=3}^{\infty}\left(12 n^{2}-18 n+4\right) p_{n}\right] r^{4}
$$

also takes on negative values. The discriminant of $Q_{2}(r)$ as a quadratic form in $r^{2}$ must be positive. Therefore we have the inequality

$$
\sum_{n=3}^{\infty}\left(6 n^{2}-9 n+2\right) p_{n}<\left(9 p_{2}^{2}-10 p_{2}+1\right) / 2
$$

and the result that $p_{2}$ is less than $1 / 9$. Applying Lemma 1 , we see that

$$
\sum_{n=3}^{\infty}\left|a_{n}\right|<\sqrt{S\left(9 p_{2}^{2}-10 p_{2}+1\right) / 2}
$$

with

$$
S=\sum_{n=3}^{\infty} 1 /\left(6 n^{2}-9 n+2\right)
$$

By use of Lemma 2, we have

$$
\sum_{n=2}^{\infty}\left|a_{n}\right|<\sqrt{p_{2}}+\sqrt{0.04752} \sqrt{9 p_{2}^{2}-10 p_{2}+1}
$$

and, by use of Lemma 3, we have

$$
\sum_{n=2}^{\infty}\left|a_{n}\right|<\sqrt{2}-1 .
$$

Applying Rouche's Theorem to the function 


$$
g(z)=1+\sqrt{2} z+\sum_{n=2}^{\infty} a_{n} z^{n}
$$

we see that, if the function $g(z)$ is analytic in the circle $|z| \leq 1$, then $g(z)$ has exactly one zero within this circle, and therefore that $g(z)$ is not the square of an analytic function in this circle. Since the convexity of the mean must break down only for values of $r$ greater than one, we have established the theorem.

5. Examples of nonconvex means. Let $f(z)$ be a single-valued branch of the function $\left[(1-z)^{2} /(1-\epsilon z)\right]^{2 / t}$, with $\epsilon=0.19$. We shall show that if $t \geq 5.66$, then the mean $\mathbb{M}_{t}(r ; f)$ is not convex in $r<1$. Since

$$
[f(z)]^{t / 2}=1+(-2+\epsilon) z+\left[(1-\epsilon)^{2} z^{2} /(1-\epsilon z)\right]
$$

it follows that

$$
\mathbb{M}_{t}(r ; f)=[h(r)]^{1 / t} \text {, }
$$

with

$$
h(r)=1+\left(4-4 \epsilon+\epsilon^{2}\right) r^{2}+\left[(1-\epsilon)^{4} r^{4} /\left(1-\epsilon^{2} r^{2}\right)\right]
$$

By straight-forward calculation, we have

$$
\begin{aligned}
& (1+\epsilon) h(1)=6-2 \epsilon=5.62 ;(1+\epsilon)^{2} h^{\prime}(1)=12-4 \epsilon^{2}=11.8556 ; \\
& (1+\epsilon)^{3} h^{\prime \prime}(1)=20+4 \epsilon-4 \epsilon^{2}-4 \epsilon^{3}=20.588164 ;
\end{aligned}
$$

and

$$
\begin{aligned}
(1+\epsilon)^{4} Q(r) & =(1+\epsilon)^{4}\left[h h^{\prime \prime}-(1-1 / t)\left(h^{\prime}\right)^{2}\right] \\
& \leq(1+\epsilon)^{4}[115.71-(1-1 / t)(140.55)] \\
& <0 \text {, if } t>140.55 / 24.84, \text { and therefore if } t \geq 5.66 .
\end{aligned}
$$

Thus we have examples of nonconvex means $\mathbb{R}_{t}(r ; f)$ for $t \geqq 5.66$ even under the restriction that $f(z)$ does not vanish in its circle of analyticity.

\section{References}

1. E. F. Beckenbach, W. Gustin, and H. Shniad, On the mean modulus of an analytic function, Bull. Amer. Math. Soc. 55 (1949), 184-190. 
2. Glaisher, Tables, Quarterly Journal of Pure and Applied Mathematics, 45, 1914, page 148 .

3. G. H. Hardy, The mean value of the modulus of an a.ralytic function, Proc. London Math. Soc. (2) 14 (1915), 269-277.

4. G. H. Hardy, J. E. Littlewood and G. Pólya, Inequalities, Cambridge, 1934.

5. G. Polya and G. Szegö, Aufgaben und Lehrsätze aus der Analysis I, Berlin, 1925.

\section{UNIVERSITY OF ARKANSAS}




\section{PACIFIC JOURNAL OF MATHEMATICS}

\section{EDITORS}

\author{
R. M. BOEINSON \\ University of California \\ Berkeley 4, California \\ E. HewitT \\ University of Washington \\ Seattle 5 , Washington
}

\section{R. P. DILWOR TH}

California Institute of Technology

Pasadena 4, California

E. F. BECKENBACH

University of California

Los Angeles 24, California

\section{ASSOCIATE EDITORS}

$\begin{array}{ll}\text { H. BUSEMANN } & \text { P. R. HALMOS } \\ \text { HERBERT FFDERER } & \text { IIEINZ HOPF } \\ \text { MARSHALL IJALL } & \text { R. D. JAMES }\end{array}$

\author{
BøRGE JESSEN \\ PAUL LÉVY \\ GEORGE PÓLYA
}

\author{
J. J. STOKER \\ E. G. STR AUS \\ KÔSAKU YOSIDA
}

\section{SFONSORS}

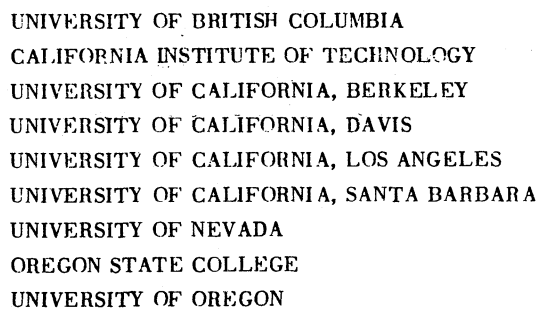

\author{
UNIVERSITY OF SOUTHERN CALIFORNIA \\ STANFORD RESEARCH INSTITUTE \\ STANFORD UNIVERSITY \\ WASHINGTON STATE COLLEGE \\ UNIVERSITY OF WASHINGTON \\ AMERICAN MATHEMATICAL SOCIETY \\ NATIONAL BUREAU OF STANDARDS, \\ INSTITUTE FOR NUMERICAL ANALYSIS
}

Mathematical papers intended for publication in the Pacific Journal of Mathematics should be typewritten (double spaced), and the author should keep a complete copy. Manuscripts may be sent to any of the editors except Robinson, whose term expires with the completion of the present volume; they might also be sent to M.M. Schiffer, Stanford University, Stanford, California, who is succeeding Robinson. All other communications to the editors should be addressed to the managing editor, E. F. Beckenbach, at the address given above.

Authors are entitled to receive 100 free reprints of their published papers and may obtain additional copies at cost.

The Pacific Journal of Mathematics is published quarterly, in March, June, September, and December. The price per volume (4 numbers) is $\$ 8.00$; single issues, $\$ 2.50$. Special price to individual faculty members of supporting institutions and to individual members of the American Mathematical Society: $\$ 4.00$ per volume; single issues, $\$ 1.25$.

Subscriptions, orders for back numbers, and changes of address should be sent to the publishers, University of California Press, Berkeley 4, California.

Printed at Ann Arbor, Michigan. Entered as second class matter at the Post Office, Berkeley, California.

\section{UNIVERSITY OF CALIFORNIA PRESS • BERKELEY AND LOS ANGELES}




\section{Pacific Journal of Mathematics}

\section{Vol. 3, No. 3 \\ May, 1953}

L. Carlitz, Some theorems on generalized Dedekind sums ............ 513

L. Carlitz, The reciprocity theorem for Dedekind sums ............. 523

Edward Richard Fadell, Identifications in singular homology theory..... . . 529

Harley M. Flanders, A method of general linear frames in Riemannian

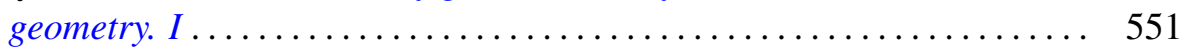

Watson Bryan Fulks, The Neumann problem for the heat equation ........ 567

Paul R. Garabedian, Orthogonal harmonic polynomials.............. 585

R. E. Greenwood and Andrew Mattei Gleason, Distribution of round-off errors for running averages .............................. 605

Arthur Eugene Livingston, The space $H^{p}, 0<p<1$, is not normable ... 613

M. N. Mikhail, On the order of the reciprocal set of a basic set of polynomials .......................................... 617

Louis Joel Mordell, On the linear independence of algebraic numbers . . . . 625

Leo Sario, Alternating method on arbitrary Riemann surfaces .......... 631

Harold Nathaniel Shapiro, Iterates of arithmetic functions and a property of the sequence of primes.............................. 647

H. Shniad, Convexity properties of integral means of analytic functions . . . . 657

Marlow C. Sholander, Plane geometries from convex plates ........... 667 